\title{
ARITHMETIC TRANSLATIONS OF AXIOM SYSTEMS
}

\author{
BY \\ HAO WANG
}

By applying the method of arithmetization to some proof of the well known Löwenheim-Skolem-Gödel theorem, we can prove that for each ordinary axiom system $\mathrm{S}$ (for example, the original Zermelo set theory as refined by Skolem) there are arithmetic predicates expressible in the notation of ordinary number theory such that when they are substituted for the predicates (for example, the membership predicate) in the axioms of $S$, the resulting assertions are all provable in the system obtained from ordinary number theory by adding the arithmetic statement $\operatorname{Con}(\mathrm{S})$ (expressing the consistency of S) as a new axiom.

With the adoption of a suitable definition for the notion of translatability, the theorem can be rephrased as saying that every system $\mathbf{S}$ is translatable into the system obtained from number theory by adding Con(S) as an axiom. As the theorem reveals the strength of the assertion Con(S) for each given system $\mathrm{S}$, it can be applied in considerations regarding problems of relative consistency. For two special systems $\mathrm{N}$ and $\mathrm{N}^{\prime}$, it is shown that $\mathrm{N}^{\prime}$ is translatable into $\mathrm{N \#} \mathrm{(namely,} \mathrm{the} \mathrm{system} \mathrm{obtained} \mathrm{from} \mathrm{N}$ by adding Con $(\mathrm{N})$ as an axiom) and that therefore the relative consistency of $\mathrm{N}^{\prime}$ to $\mathrm{N \#}$ can be proved in number theory. It is also observed that if $\mathrm{N}$ is $\omega$-consistent, then $N \#$ is consistent. The same method can be applied, for similarly related systems $S$ and $S^{\prime}$, to prove the relative consistency of $S^{\prime}$ to $S \#$, although in certain cases $S^{\prime}$ is demonstrably not translatable into $S$.

Using the same notion of translation, we have also, from Gödel's theorem on the impossibility of proving $\operatorname{Con}(\mathrm{S})$ in $\mathrm{S}$, the conclusion that if $\operatorname{Con}(\mathrm{S})$ is provable in $S^{\prime}$, then $S^{\prime}$ is not translatable into $S$. Since, as it is known, the consistency of any given system can in a certain sense be proved in a stronger system, it follows that there exists an infinite sequence of systems $\mathrm{L}_{0}$ (being the ordinary number theory), $\mathrm{L}_{1}, \mathrm{~L}_{2}, \cdots$ which are all of the same notation as the ordinary number theory and of which each is translatable into all its successors but none is translatable into any of its predecessors. There are also predicates $\mathrm{P}_{1}, \mathrm{P}_{2}, \cdots$ such that $\mathrm{P}_{m}$ is definable in $\mathrm{L}_{n}$ when and only when $m$ is not greater than $n$. The question whether the arithmetic translations of the predicates such as $\mathrm{P}_{1}, \mathrm{P}_{2}, \cdots$ could be recursive predicates seems to be an open question.

To help us in the studies reported in this paper, Professors Paul Bernays, W. V. Quine, and J. Barkley Rosser have given generously their time and made very valuable criticisms as well as suggestions.

Received by the editors October 21, 1950 and, in revised form, April 15, 1951. 
1. The notion of translatability. Let $S$ and $S^{\prime}$ be any two formal systems containing quantifiers and truth-functional connectives $\left({ }^{1}\right)$.

Definition 1. $\mathrm{S}$ is said to be translatable into or has a model in (or obtainable within or contained as a part in) $S^{\prime}$ if there exists an effective way of replacing all the predicates of $S$ by predicates of $S^{\prime}$ such that the resulting statements in place of the theorems of $S$ all are theorems of $S^{\prime}$.

More explicitly, this definition contains at least the following parts:

1.1. The translation of a quantification (namely, a formula beginning with a quantifier) of $\mathrm{S}$ is a quantification in $\mathrm{S}^{\prime}$.

1.2. The translation of the negation of a formula of $S$ is the negation of the translation of the formula.

1.3. The translation of the conjunction of two formulas of $S$ is the conjunction of their translations.

1.4. A special case of 1.2. The translation of the negation of a statement (that is, a formula containing no free individual variables) of $S$ is the negation of its translation.

1.5. The translations of the theorems of $\mathrm{S}$ all are theorems of $\mathrm{S}^{\prime}$. Or alternatively,

1.5.1. The translations of the axioms of $S$ are theorems of $S^{\prime}$.

1.5.2. If a statement of $S$ is deducible from certain other statements of $S$ by a rule of inference and the translations of these latter statements of $S$ are theorems of $S^{\prime}$, then the translation of the former statement is also a theorem of $\mathrm{S}^{\prime}$.

It appears that in most cases where we tend to say that a system $S$ is translatable into or obtainable within a system $S^{\prime}$, the conditions in the above definition are satisfied. For example, this is the case when we say that arithmetic is obtainable in set theory, or that the simple theory of types is translatable into Zermelo set theory. Nevertheless, for purposes of metamathematical considerations, it seems usually sufficient to use the following simpler and more comprehensive definition $\left({ }^{2}\right)$.

Definition 2. $S$ is said to be translatable into $S^{\prime}$ if there exists a (general) recursive function $T$ mapping the set of numbers representing (via an arithmetization of the syntax) the statements of $S$ into the set of numbers representing those of $S^{\prime}$ such that the set of (the numbers representing) the theorems of $\mathbf{S}$ is mapped into the set of those of $\mathbf{S}^{\prime}$ and the image of the

(1) Functions or functors are not mentioned in the definition because it is known that theoretically they can always be replaced by predicates; see p. 460 of Hilbert and Bernays, Grundlagen der Mathematik, vol. 1, Berlin, 1934.

(2) It is not important that Definition 2, unlike Definition 1, is phrased in terms of an arithmetized syntax. A reformulation of Definition 1 in similar terms is also possible, although it would be somewhat lengthy.

Incidentally, according to either definition, the relation between $S$ and $S^{\prime}$ of being translatable into each other is obviously an equivalence relation, being reflexive, transitive, and symmetric. 
negation of a statement is the negation of the image of the statement.

It is easy to see that if $S$ is translatable into $S^{\prime}$ according to Definition 1 , it is so also according to Definition 2, but not conversely. One example where $S$ is translatable into $S^{\prime}$ according to Definition 2 but not according to Definition 1 is the case where $S$ is the quantification theory (the first-order predicate calculus) and $S^{\prime}$ is the theory of truth functions (the propositional calculus) $\left.{ }^{3}\right)$. If $S$ is translatable into $S^{\prime}$ according to Definition 2, we can only conclude that 1.4-1.5 (but not necessarily 1.1-1.3) hold. Notwithstanding the difference between these two definitions, the theorems in this paper are not affected whether we use one or the other definition. Therefore, in reading this paper either definition can be assumed according to one's preference.

When two apparently different systems are translatable in to each other, we often say that they are essentially of equal strength. Thus, in a certain sense, those properties of a system which remains unchanged under translations constitute its essence while the other properties are theoretically less important. If we stick to the first definition, we might also say that it is the structure of the system that is mathematically important, not its contents.

Definition 3. Two systems $S$ and $S^{\prime}$ are said to be of equal strength if they are translatable into each other. $S^{\prime}$ is said to be stronger than $S$ if $S$ is translatable into $S^{\prime}$ but not conversely.

A terminological remark is here in order. Throughout this paper, by number theory or ordinary number theory is meant the system $Z$ of Hilbert and Bernays $\left({ }^{4}\right)$ which contains, besides quantification theory, the theory of identity, the Peano axioms, and the recursive equations for addition and multiplication. The theoretically dispensable $\mu$-operators (the smallest number such that) can also be added, together with the basic properties of these operators.

THEOREM 1. If $\mathrm{S}$ is translatable into $\mathrm{S}^{\prime}$ then $\mathrm{S}$ is consistent if $\mathrm{S}^{\prime}$ is, and $\mathrm{Con}(\mathrm{S})$ is derivable from $\mathrm{Con}\left(\mathrm{S}^{\prime}\right)$ in number theory.

Proof. If - Con(S), then there is a statement of $S$ such that both it and its negation are theorems of S. By 1.5, the translations of these two statements are theorems of $S^{\prime}$. But by 1.4 , the translation of the negation is the negation of the translation. Hence, $-\operatorname{Con}\left(\mathrm{S}^{\prime}\right)$. Therefore, if $\operatorname{Con}\left(\mathrm{S}^{\prime}\right)$ then Con $(\mathrm{S})$. Moreover, the derivation can be carried out in number theory, since we assume the mapping to be effective.

Definition 4. A system which contains number theory as a part is said to be a mathematical system. (In particular, the system $Z$ of number theory is a mathematical system.)

(3) Such a translation is obtainable by omitting the quantifiers and the individual variables in every formula of the quantification theory. Cf. p. 70 of Hilbert and Ackermann, Grundz ïge der theoretischen Logik, 2d ed., Berlin, 1938.

(4) Hilbert and Bernays, op. cit., p. 371. 
According to Gödel's theorem on consistency proofs, we have:

1.6. If $\mathrm{S}$ is a consistent mathematical system, $\operatorname{Con}(\mathrm{S})$ is not provable in $S$ nor in number theory.

THEOREM 2. If $\mathrm{S}^{\prime}$ contains a consistent mathematical system $\mathrm{S}$ as a part and Con $(\mathrm{S})$ is a theorem of $\mathrm{S}^{\prime}$, then $\mathrm{S}^{\prime}$ is stronger than $\mathrm{S}$.

Proof. By hypothesis, $\mathrm{S}^{\prime}$ contains number theory as a part. Therefore, by Theorem 1 , if $\mathrm{S}^{\prime}$ were translatable into $\mathrm{S}, \mathrm{Con}\left(\mathrm{S}^{\prime}\right)$ would be derivable in $\mathrm{S}^{\prime}$ from Con $(S)$. Since $\operatorname{Con}(S)$ is by hypothesis provable in $S^{\prime}$, Con $\left(S^{\prime}\right)$ would then be provable in $\mathrm{S}^{\prime}$. Hence, by $1.6, \mathrm{~S}^{\prime}$ would be inconsistent. Therefore, by Theorem 1, if $S^{\prime}$ were translatable into $S, S$ would also be inconsistent. But $S$ is consistent by hypothesis. Hence, $S^{\prime}$ is not translatable into $S$ and, by Definition $3, \mathrm{~S}^{\prime}$ is stronger than $\mathrm{S}$.

Thus, for example, each set theory which contains the number theory $Z$ as a part and in which $\operatorname{Con}(Z)$ is a theorem is stronger than $Z$ (that is, not translatable into $Z$ ).

Let $Z_{\mathrm{S}}$ be the system obtained from $Z$ by adding $\operatorname{Con}(\mathrm{S})$ as an axiom. Then we can prove:

TheOREM 3. If $Z_{\mathrm{S}}$ is consistent, then $\mathrm{S}$ is consistent.

Proof. If $\mathrm{S}$ is inconsistent, then the arithmetization of the proof of a contradiction in $S$ gives a numerical counter example to $C o n(S)$. This numerical counter example is available in $Z$, so that $Z_{\mathrm{S}}$ is inconsistent.

On the other hand, we have:

THEOREM 4. If $\mathrm{S}$ is consistent and $\mathrm{Z}$ is w-consistent, then $Z_{\mathrm{S}}$ is consistent.

Proof. Let $\operatorname{Pr}(m, n)$ be the recursive arithmetic predicate expressing that $m$ is the number of a proof in $\mathrm{S}$ for the statement whose number is $n$. If $n_{0}$ is the number of a statement (for example, $0=1$ ) whose negation is provable in $\mathrm{S}$, then for every fixed number $m_{0},-\operatorname{Pr}\left(m_{0}, n_{0}\right)$ is "true" (or verifiable) because $S$ is consistent. Since $\operatorname{Pr}$ is recursive, $-\operatorname{Pr}\left(m_{0}, n_{0}\right)$ is therefore for every fixed $m_{0}$ provable in $Z$. By hypothesis, $Z$ is $\omega$-consistent. Therefore, $Z$ remains consistent if we add $(m)-\operatorname{Pr}\left(m, n_{0}\right)$ as a new axiom. Hence, $Z_{\mathbb{S}}$ is consistent.

If $S$ happens to be a system in which the $\omega$-consistency of $Z$ can be proved, then we seem to be able to infer that if $S$ is consistent, then $Z_{S}$ is consistent.

By applying the method of arithmetization to either Gödel's proof or Henkin's proof of the strengthened Löwenheim theorem, we can prove the following theorem:

THEOREM 5. If $\mathrm{S}$ is consistent, then we can define in the system $Z_{\mathrm{S}}$ certain predicates such that the axioms $\mathrm{A}_{1}, \mathrm{~A}_{2}, \cdots$ of $\mathrm{S}$ all become provable in $Z_{\mathrm{S}}$ if in them we replace all the predicates one by one by these predicates defined in $Z_{S}$ and let all variables range over natural numbers. 
At first we proved this theorem as a slight extension of a result of Hilbert and Bernays $\left({ }^{5}\right)$ obtained by arithmetizing a variant of Gödel's proof of the completeness of quantification theory. Both Professor Rosser and the referee found our proof too complex and suggested arithmetizing instead the much simpler Henkin proof $\left({ }^{6}\right)$. We quote their suggestions:

What must be done is to write the Henkin proof in terms of definition by induction. For this, we modify the Henkin proof slightly $\left({ }^{7}\right)$. Starting with $\Lambda$, we let $u_{i}$ be symbols not occurring among the symbols of $S_{0}$. We also enumerate all formulas $\mathrm{C}_{0}, \mathrm{C}_{1}, \cdots$, as follows: If $n$ is even, say $n=2 m$, then pick the first formula $(\exists x) F(x)$ deducible from $\Lambda$ together with $\mathrm{C}_{0}, \mathrm{C}_{1}, \cdots, \mathrm{C}_{n-1}$, and such that $(\exists x) F(x)$ was not formerly picked in defining a $\mathrm{C}_{r}$, and take $\mathrm{C}_{n}$ to be $F\left(u_{m}\right)$. If $n$ is odd, say $n=2 m+1$, take $\mathrm{C}_{n}$ to be the first formula which is not already a $C_{r}$, and which involves only $u_{1}$, $u_{2}, \cdots, u_{m}$ together with symbols of $S_{0}$, and such that no contradiction can be derived from $\Lambda$ together with $\mathrm{C}_{0}, \mathrm{C}_{1}, \cdots, \mathrm{C}_{n}$. Then $\Gamma_{\omega}$ can be taken as the set of all $\mathrm{C}_{n}$ 's, and Henkin's proof easily goes through in number theory. From this result, the above Theorem 5 follows immediately.

We have not attempted to work out the above suggestions because Dr. Gisbert Hasenjaeger had already obtained with a similar modification of Henkin's proof $\left({ }^{8}\right)$ a stronger result which contains Theorem 5 as a part, before we received the above suggestions. However, these circumstances seem to make it both justifiable and desirable to omit from this paper our earlier complex proof of the theorem above. Accordingly, we shall assume Theorem 5 , but present here no detailed proof for it.

By Definition 1, Definition 2, and Theorem 5, we have:

THEOREM 6, Every system $\mathrm{S}$ is translatable into its corresponding system $Z_{\mathrm{S}}$. Moreover, there is an effective method by which, given any system $\mathrm{S}$, we can find a translation which translates it into $\mathrm{Z}_{\mathrm{s}}$.

2. Proofs of relative consistency. To illustrate the applications of Theorem 6 , we consider two special systems $\mathrm{N}$ and $\mathrm{N}^{\prime}$ such that $\mathrm{N}^{\prime}$ is related to $\mathrm{N}$ as an $(n+1)$ th order predicate calculus is to an $n$th except that variables of the $(n+1)$ th type are not used in defining classes of lower types. It is proved that $\mathrm{N}^{\prime}$ is translatable into the system obtained from $\mathrm{N}$ by adding Con $(\mathrm{N})$ as an axiom.

(5) See especially pp. 185-188 and pp. 234-352 of Hilbert and Bernays, ibid., vol. 2, 1939. Compare also Theorem 5 and footnote 3 of Remarks on the comparison of axiom systems, Proc. Nat. Acad. Sci. U.S.A. vol. 36 (1950) pp. 448-453. Certain definitions and theorems of this paper have been summarized there, and a few more references have also been given there.

(6) J. Symbolic Logic vol. 14 (1949) pp. 159-166.

(7) See pp. 162-163, ibid.

(8) In a report for Professor Bernays's seminar at Zurich, Dr. Hasenjaeger presented in the winter of 1950 substantially the same simplification of Henkin's proof as sketched above, although he did not discuss the question of arithmetization then. 
$\mathrm{N}$ is a weak system of set theory formulated after the manner of Quine and belonging to the kind of set theory in which the distinction between elements (or sets) and (non-element) classes is made so that all classes are classes of elements. The axioms of $\mathrm{N}$ assure us only that the null class is an element and classes of one or two members are elements. Accordingly $\mathrm{N}$ admits a model containing denumerably many elements plus classes of them. Such a model seems to make it clear that $\mathrm{N}$ is roughly as strong as a second-order predicate calculus founded on natural numbers.

$\mathrm{N}$ may also be characterized as a system obtained from a well-developed system of Quine $\left.{ }^{9}\right)$ by replacing all his elementhood axioms by a single one stating that pairs are elements. It is formulated within the quantification theory $Q$ used ordinarily, with a single special predicate written as $\epsilon$.

In $\mathrm{N}$, element variables $a, b, c$, and so on are introduced by contextual definitions such as:

$$
\text { (a)A(a) for } \quad(x)((\exists y)(x \in y) \supset A(x)) .
$$

2.2 .

$(\exists a)$ for $-(a)$ -

And identity is defined in the usual manner:

$$
x=y \text { for }(z)(z \in x \equiv z \in y) .
$$

The special axioms of $\mathrm{N}$ are given in $\mathrm{C} 1-\mathrm{C} 3$.

C1. $x=y \supset(x \in z \supset y \in z)$.

C2. $(\exists a)(b)(b \in a \equiv(b=x \vee b=y))$.

C3. If $A$ is any formula of $\mathrm{N}$ in which $y$ is not free, then $(\exists y)(a)(a \in y$ $\equiv \mathrm{A})$.

It is known that the number theory $Z$ is translatable into $N$. One way of making the translation is as follows $\left({ }^{10}\right)$. Identify 0 with the null class and the successor of a natural number with its unit class. In this way, all natural numbers are identified with elements of $\mathrm{N}$. Then define the class $\mathrm{Nn}$ of natural numbers as the intersection of all classes $x$ of $\mathrm{N}$ such that $x$ contains 0 and, for every $n$, if $x$ contains $n$ it also contains the successor of $n$. The principle of induction follows immediately: Every class which contains $\mathbf{0}$ and contains the successor of $n$ if containing $n$, contains Nn. Variables $m, n$, and so on which take natural numbers as values and the $\mu$-operators $\mu_{m}, \mu_{n}$, and so on can be introduced in $\mathrm{N}$ by contextual definitions. The following metatheorems of $\mathrm{N}$ are provable.

2.4. If $y$ is not free in $\mathrm{A}$, then $(\exists y)(m)(m \in y \equiv A)$.

2.5. ( $\exists m) A(m) \supset A\left(\mu_{m} A(m)\right)$.

2.6. $(n)(A(n) \equiv B(n)) \supset \mu_{n} A(n)=\mu_{n} B(n)$.

The system $\mathrm{N}^{\prime}$ is an extension of $\mathrm{N}$, the primitive notations of which being those of $\mathrm{N}$ plus a new kind of variable $X, Y$, and so on and a new dyadic

( $\left.{ }^{9}\right)$ Mathematical logic, New York, 1940.

(10) See J. Symbolic Logic vol. 13 (1948) pp. 129-137. 
predicate $\eta$. The atomic formulas of $\mathrm{N}^{\prime}$ are $x \in y$, and so on, and $x \eta Y$, and so on from which we build up all the formulas of $\mathrm{N}^{\prime}$ by truth-functional connectives and quantifiers. $\mathrm{N}^{\prime}$ contains the quantification theory for the variables $x, y$, and so on, and that for the variables $X, Y$, and so on. The special axioms of $\mathrm{N}^{\prime}$ are $\mathrm{C} 1-\mathrm{C} 3$ and the following additional ones $\left({ }^{11}\right)$.

C4. If $\mathrm{A}$ is a formula of $\mathrm{N}^{\prime}$ in which $Y$ is not free, then $(\exists Y)(x)(x \eta Y \equiv \mathrm{A})$.

C5. $(x)(x \in y \equiv x \in z) \supset(y \eta X \supset z \eta X)$.

Let us assume that the syntax of $\mathrm{N}$ and that of $\mathrm{N}^{\prime}$ have both been arithmetized in the usual manner. Since $N$ is a part of $N^{\prime}$, let us assume that the arithmetization of the syntax of $\mathrm{N}$ coincides with a part of that of $\mathrm{N}^{\prime}$. With such arithmetizations, we have two arithmetic statements Con(N) and $\operatorname{Con}\left(\mathrm{N}^{\prime}\right)$ expressing respectively that $\mathrm{N}$ is consistent and that $\mathrm{N}^{\prime}$ is consistent. Let $\mathrm{N} \#$ be the system obtained from $\mathrm{N}$ by adding Con $(\mathrm{N})$ as a new axiom. Then we can also obtain an arithmetization of the syntax of N\# from that of $\mathrm{N}$ and, therefore, there is an arithmetic statement Con $(\mathrm{N \# )}$

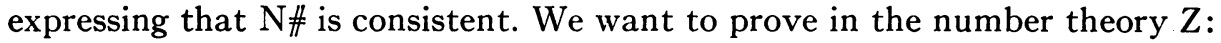
If $\operatorname{Con}(\mathrm{N} \#)$, then $\operatorname{Con}\left(\mathrm{N}^{\prime}\right)$.

This we do in the following manner. By Theorem $6, \mathrm{~N}$ is translatable into the system $Z_{\mathrm{N}}$ obtained from number theory by adding $\operatorname{Con}(\mathrm{N})$ as a new axiom. More precisely, we know from Theorem 5 that in $Z_{\mathrm{N}}$ theorems can be proved which are like the axioms of $\mathrm{N}$ given in $\mathrm{C} 1-\mathrm{C} 3$ (imagining that all the defined symbols $=, a, b$, and so on have been eliminated with the help of the defintions 2.1-2.3) except for containing an arithmetic predicate $\in^{*}$ (say) of $Z_{\mathrm{N}}$ in place of $\in$ and variables $m, n$, and so on instead of the variables $x, y$, and so on. Since $\mathrm{N}$ contains $Z, \mathrm{~N} \#$ contains $Z_{\mathrm{N}}$. Therefore, these arithmetic translations of the axioms $\mathrm{C} 1-\mathrm{C} 3$ of $\mathrm{N}$ and $\mathrm{N}^{\prime}$ are all provable in $\mathrm{N \# .}$ For example, corresponding to $\mathrm{C} 1$, we can prove in $\mathrm{N \# :}$

$\mathrm{C1}^{\prime} .(m)\left(m \in{ }^{*} k \equiv m \in{ }^{*} j\right) \supset\left(k \in{ }^{*} n \supset j \in{ }^{*} n\right)$.

Let us use $m^{*}, k^{*}$, and so on, as abbreviations $\left({ }^{12}\right)$ :

2.7. $m^{*}$ for $\mu_{n}(j)\left(j \in \in^{*} n \equiv j \in{ }^{*} m\right)$, and so on.

Then we have in $\mathrm{N \# :}$

2.8. $j \in{ }^{*} m^{*} \equiv j \in{ }^{*} m$.

Proof. Since $(j)\left(j \in \in^{*} m \equiv j \in^{*} m\right)$, so $(\exists n)(j)\left(j \in{ }^{*} n \equiv j \in^{*} m\right)$. By 2.5 and 2.7 , the theorem follows immediately.

Let $\mathrm{A}$ be an arbitrary formula of $\mathrm{N \#} \mathrm{built} \mathrm{up} \mathrm{exclusively} \mathrm{from} \mathrm{formulas}$ of the forms $n \in{ }^{*} m$ and $m^{*} \in y$ with the help of quantifiers and truth-functional connectives. By 2.4 and 2.8 , we have:

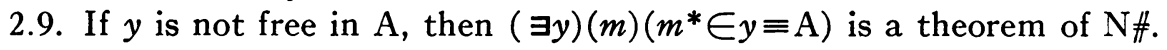

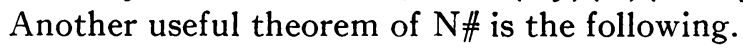

2.10. $(j)\left(j \in{ }^{*} k \equiv j \in{ }^{*} m\right) \supset\left(k^{*} \in y \supset m^{*} \in y\right)$.

Proof. Assume: $(j)\left(j \in{ }^{*} k \equiv j \in{ }^{*} m\right)$. Therefore, $\quad(j)\left(j \in{ }^{*} n \equiv j \in{ }^{*} k\right)$

(11) In $\mathrm{N}^{\prime}, \mathrm{C} 1$ becomes redundant on account of the presence of $\mathrm{C} 4$ and $\mathrm{C5}$.

(12) This differs only slightly from a device which Professors Bernays and Rosser suggested to us to ensure that we can prove in $\mathrm{N} \#$ the counterpart of $\mathrm{C} 5$. 
$\equiv(j)\left(j \in^{*} n \equiv j \in^{*} m\right)$. Hence, by 2.6 and $2.7, k^{*}=m^{*}$. Hence, $k^{*} \in y \supset m^{*} \in y$, and 2.10 is proved.

To show that the whole system $\mathrm{N}^{\prime}$ is translatable into the system N\#, we use a translation according to which the translation in $\mathrm{N \#} \mathrm{of} \mathrm{a} \mathrm{statement}$ A of $\mathrm{N}^{\prime}$ is obtained from A by the following transformations: (1) Any formula of $\mathrm{N}^{\prime}$ of the form $x \in y$ is replaced by a formula of $\mathrm{N}^{\prime}$ of the form $m \in{ }^{*} k$, and the associated quantifiers $(x)$ and $(y)$ are replaced by the quantifiers $(m)$ and $(k)$; (2) Any formula of $\mathrm{N}^{\prime}$ of the form $x \eta Y$ is replaced by a formula of $\mathrm{N}^{\prime}$ of the form $m^{*} \in y$, and the associated quantifiers $(x)$ and $(Y)$ are replaced by $(m)$ and $(y) ;(3)$ Every truth-functional connective remains unchanged.

It should be easy to see that the translation satisfies all the conditions in Definition 1 and Definition 2. The only item that calls for some comment seems to be 1.5 , which requires that all theorems of $\mathrm{N}^{\prime}$ be translated into theorems of $\mathrm{N \# .}$

As mentioned above, the translations of $\mathrm{C} 1-\mathrm{C} 3$ are all theorems of $\mathrm{N \#}$. And the translations of $\mathrm{C} 4$ and $\mathrm{C} 5$ are just 2.9 and 2.10 which have been proved for N\#. Moreover, we can obtain in N\# the quantification theory for the variables $x, y$, and so on and that for the variables $m, n$, and so on. Hence, all theorems of $\mathrm{N}^{\prime}$ are transformed into theorems of $\mathrm{N \#} \mathrm{according} \mathrm{to} \mathrm{the}$ translation described above.

\section{Theorem 7. $\mathrm{N}^{\prime}$ is translatable into $\mathrm{N \# .}$}

Hence, by Theorem 1, we have:

2.11. $\operatorname{Con}\left(\mathrm{N}^{\prime}\right)$ is derivable from $\operatorname{Con}\left(\mathrm{N \# )}\right.$ in number theory; $\operatorname{Con}\left(\mathrm{N}^{\prime}\right)$ is derivable from $\operatorname{Con}\left(\mathrm{N \# )}\right.$ in $\mathrm{N}^{\prime}$.

Therefore, by 1.6 , we have:

2.12. If $\mathrm{N}^{\prime}$ is consistent, then $\operatorname{Con}\left(\mathrm{N \# )}\right.$ is not a theorem of $\mathrm{N}^{\prime}$. In other words, if $\operatorname{Con}\left(\mathrm{N \# )}\right.$ is a theorem of $\mathrm{N}^{\prime}$, then $\mathrm{N}^{\prime}$ is inconsistent.

An interesting connection between $\mathrm{N}$ and $\mathrm{N \#}$ is embodied in the next theorem $\left({ }^{13}\right)$.

THEOREM 8. If $\mathrm{N}$ is $\omega$-consistent, then $\mathrm{N \#} \mathrm{is} \mathrm{consistent.}$

Proof. Assume that N\# is inconsistent. Then every statement of N\#, in-

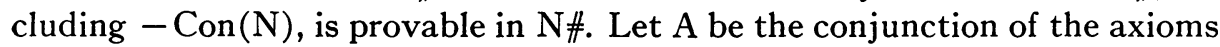
of $\mathrm{N}$ used in the proof for $-\operatorname{Con}(\mathrm{N})$. Then, since $\operatorname{Con}(\mathrm{N})$ is the only axiom of $\mathrm{N} \#$ which is not also an axiom of $\mathrm{N}$, we have a proof for $-\mathrm{Con}(\mathrm{N})$ in $\mathrm{N} \#$ using the conjunction $(\mathrm{A} \& \mathrm{Con}(\mathrm{N}))$. Therefore, by the deduction theorem, we can prove $((\mathrm{A} \& \operatorname{Con}(\mathrm{N})) \supset-\operatorname{Con}(\mathrm{N}))$ in the quantification theory of $\mathrm{N}$. Therefore by truth-functional transformations, (A $\supset-\operatorname{Con}(\mathrm{N}))$ is a theorem of the quantification theory too. Hence, $-\mathrm{Con}(\mathrm{N})$ is a theorem of $\mathrm{N}$.

Therefore, if $\mathrm{P} c(n)$ is the arithmetic predicate expressing that $n$ repre-

${ }^{(13)}$ This theorem was communicated to us by Professors Rosser and Bernays. 
sents a proof in $\mathrm{N}$ for the statement (say) $0=1$, then $(\exists n) \mathrm{P} c(n)$ is a theorem of $\mathrm{N}$. If $\mathrm{N}$ is inconsistent, then a fortiori it is also $\omega$-inconsistent. On the other hand, if $\mathrm{N}$ is consistent then, for every given $n,-\mathrm{Pc}(n)$ is true and therefore provable in $\mathrm{N}$, because it is a statement obtained from a primitive recursive predicate by substituting a constant numeral in the argument and every true statement of such a form is provable in number theory. But $(\exists n) \operatorname{Pc}(n)$ is also a theorem of $\mathrm{N}$. Therefore, $\mathrm{N}$ is $\omega$-inconsistent. Combining

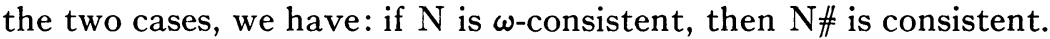

Therefore, by 2.11 , we have:

2.13. If $\mathrm{N}$ is $\omega$-consistent, then $\mathrm{N}^{\prime}$ is consistent.

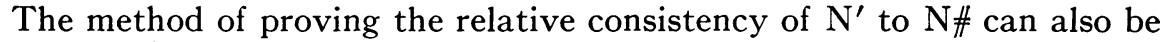
applied to other systems. Let $\mathrm{S}$ be the part of Zermelo set theory consisting of the Aussonderungsaxiom, the axiom of extensionality, the axiom of infinity, and the axiom of power set; let $S^{\prime}$ be a simple theory of types with $S$ as its theory of individuals. Since, according to a result of Quine $\left({ }^{14}\right)$, the simple theory of types founded on natural numbers is translatable into $\mathrm{S}$, we can apply the method of proving the relative consistency of $N^{\prime}$ to $N \#$ and prove:

2.14. If $\mathrm{S}$ is $\omega$-consistent, then $\mathrm{S}^{\prime}$ is consistent.

Other examples may be given. Consider, for example, the simple theory of types as formulated by Gödel($\left.{ }^{15}\right)$. This system $\mathrm{P}$ is founded on natural numbers and contains, besides the Peano axioms and the axiom of extensionality, only the axiom of comprehension: If $n$ is a positive integer and A is any formula of $\mathrm{P}$ in which $y_{n+1}$ is not free, then $\left(\exists y_{n+1}\right)\left(x_{n}\right)\left(x_{n} \in y_{n+1} \equiv \mathrm{A}\right)$ is an axiom of $P$. It is easy to see that this can be replaced by the next two principles which at first sight appear to be weaker: (1) If A contains no variable of any type higher than $n+1$, then $\left(\exists y_{n+1}\right)\left(x_{n}\right)\left(x_{n} \in y_{n+1} \equiv \mathrm{A}\right)$; (2) If $\mathrm{A}$ is any formula of $\mathrm{P}$ in which $y_{2}$ is not free, then $\left(\exists y_{2}\right)\left(x_{1}\right)\left(x_{1} \in y_{2} \equiv \mathrm{A}\right)$. Let $\mathrm{P}^{\prime}$ be the system obtained from $\mathrm{P}$ by omitting the principle (2), and let us refer to it as the monotonic simple theory of types. Then we can speak of the part $\mathrm{T}_{n}^{\prime}$ of $\mathrm{P}^{\prime}$ which contains nothing of any type higher than $n$ as the monotonic predicate calculus of the $n$th order, in contrast with the corresponding part $\mathrm{T}_{n}$ of $\mathrm{P}$ which forms the $n$th order predicate calculus. Using the method for $\mathrm{N}^{\prime}$ and $\mathrm{N}$, we can prove:

THEOREM 9. If $\mathrm{T}_{2}^{\prime}$ is $\omega$-consistent, then $\mathrm{T}_{3}^{\prime}$ is consistent; if $\mathrm{T}_{3}^{\prime}$ is $\omega$-consistent, then $\mathrm{T}_{5}^{\prime}$ is consistent; if $\mathrm{T}_{4}^{\prime}$ is $\omega$-consistent, then $\mathrm{T}_{7}^{\prime}$ is consistent; and so on.

If we call a system regular when we know that it is either inconsistent or $\omega$-consistent but that it cannot be consistent yet $\omega$-inconsistent, we obtain the next theorem.

(14) See J. Symbolic Logic vol. 1 (1936) pp. 45-57. The special systems which Quine considers are somewhat different. However, his method applies to the present case too.

(15) See Monatshefte für Mathematik und Physik vol. 38 (1931) pp. 176-178. 
2.15. If $T_{2}^{\prime}$ is consistent and $T_{2}^{\prime}, T_{3}^{\prime}, \cdots$ are all regular, then $T_{3}^{\prime}$, $\mathrm{T}_{4}^{\prime}, \cdots$, as well as $\mathrm{P}^{\prime}$, are all consistent.

Thus, in a certain sense, we can say that the reason why a predicate calculus (founded on natural numbers) of a higher order is stronger than one of a lower order merely comes from the presence of the principle (2) which enables us to form new classes of natural numbers in the predicate calculus of higher order.

The method does not seem to be applicable to the systems $T_{3}, T_{4}, \cdots$ of predicate calculi in any analogous manner. Indeed, the principle (2) seems to make much difference. For example, it is not obvious whether $\mathrm{T}_{3}$, might be translatable into $\mathrm{P}^{\prime}$.

3. Definability and translatability. It is known $\left({ }^{16}\right)$ that there exists a sequence of systems (set theories) $S_{1}, S_{2}, \cdots$ such that each contains the number theory $Z$ as well as all its predecessors, and that in each the consistency of all its predecessors can be proved. For example, the systems $T_{2}, T_{3}, \cdots$ just mentioned or certain systems respectively of similar strength can be taken as the systems $S_{1}, S_{2}, \cdots$ respectively. Hence, by Theorem 2, we have:

3.1. There exists a sequence of systems $S_{1}, S_{2}, \ldots$ such that they are all stronger than $Z$, and that if they are consistent then for every $m$ and $n, \mathrm{~S}_{m}$ is stronger than $S_{n}$ when and only when $m$ is greater than $n$.

As we know, the set theories $S_{1}, S_{2}, \cdots$ can be so formulated that in the axioms of each system merely a single dyadic predicate (the membership predicate) occurs. Let these predicates be $E_{1}, E_{2}, \cdots$, and their arithmetic translations according to Theorem 5 be $\mathrm{E}_{1}^{*}, \mathrm{E}_{2}^{*}, \cdots$, respectively.

Definition 5. A predicate $\mathrm{E}$ implicitly determined by certain assertions concerning it is said to be definable or has a model in a system $S^{\prime}$ if there exists a system $S$ which includes the assertions about $E$ as theorems and which is translatable into $\mathrm{S}^{\prime}$.

For example, the predicate of being a natural number is according to this definition definable in the system $\mathrm{N}$ described above, if we take the Peano axioms as determining the predicate. By 3.1, we have:

3.2. None of the predicates $E_{1}, E_{2}, \cdots$ is definable in $Z$. If $S_{1}, S_{2}, \cdots$ are all consistent, then for every $m$ and $n, \mathrm{E}_{m}$ is definable in $\mathrm{S}_{n}$ when and only when $m$ is not greater than $n$.

When a predicate $E$ is determined by certain postulates, a translation $\mathrm{E}^{*}$ of the predicate is determined by the translations of the postulates. Therefore, we have also:

3.3. None of the predicates $E_{1}^{*}, E_{2}^{*}, \cdots$ is definable in $Z$.

Let $Z_{\mathrm{S}}$ be, as before, the system obtained from $Z$ by adding $\operatorname{Con}(\mathrm{S})$ as a new axiom. Using the same sequence of systems, we have:

(16) Cf. Tarski's assertions on the lower half of p. 110 of J. Symbolic Logic vol. 4 (1939), as well as those on p. 318, p. 359, and p. 400 of Studia Philosophia vol. 1 (1935). 
3.4. If $Z$ is $\omega$-consistent and $S_{1}, S_{2}, \cdots$ are all consistent, then:

(a) All the systems $Z_{Z}, Z_{\mathrm{s}_{1}}, Z_{\mathrm{S}_{2}}, \cdots$ are consistent and have the same primitive notation as $Z$;

(b) Each system in the sequence $Z, Z_{\mathrm{Z}}, \mathrm{S}_{1}, Z_{\mathrm{S}_{1}}, \mathrm{~S}_{2}, Z_{\mathrm{S}_{2}}, \cdots$ is translatable into every later system in the sequence;

(c) For all positive integers $m$ and $n$, if $m-1$ is greater than $n$, then $S_{m}$ is not translatable into $Z_{\mathrm{S}_{n}}$;

(d) If $m$ is not greater than $n$, then $\mathrm{E}_{m}$ is definable in $Z_{\mathbb{S}_{n}}$, if $m-1$ is greater than $n$ then $\mathrm{E}_{m}$ is not definable in $Z_{\mathbf{S}_{n}}$;

(e) If $m-1$ is greater than $n$, then $Z_{\mathbf{S}_{m}}$ is not translatable into $Z_{\mathrm{s}_{n}}$ and, therefore, for example, each system in the sequence $Z, Z_{S_{1}}, Z_{S_{3}}, Z_{S_{5}}, \cdots$ is stronger than all its predecessors.

Proof. (a) By Theorem 4.

(b) By Theorem 5 , each $S_{i}$ is translatable into $Z_{S_{i}}$. But each $S_{i}$ contains $Z$ and $\operatorname{Con}\left(S_{i-1}\right)$ as a theorem. Hence, $Z_{S_{i-1}}$ is translatable into $S_{i}$.

(c) Since $m-1$ is greater than $n, Z_{S_{n}}$ is, by (b), translatable into $S_{m-1}$. Therefore, if $S_{m}$ were translatable into $Z_{S_{n}}, S_{m}$ would be translatable into $\mathrm{S}_{m-1}$.

(d) By Definition 5, (b), and (c).

(e) By (b) and (c).

Two questions remain unanswered: Is $Z_{\mathrm{s}_{n}}$ translatable into $S_{n}$ ? Is $Z_{\mathrm{S}_{n+1}}$ translatable into $Z_{\mathbf{S}_{n}}$ ? We know merely that $Z_{S_{n}}$ is translatable into $S_{n}$ for every $n$, if and only if, for every $n, Z_{\mathrm{S}_{n+1}}$ is not translatable into $Z_{\mathrm{S}_{n}}$.

From (d) and (e) of 3.4, we have the following corollary $\left({ }^{17}\right)$.

THEOREM 10. There exists a sequence of systems $\mathrm{L}_{0}(=\mathrm{Z}), \mathrm{L}_{1}, \mathrm{~L}_{2}, \cdots$ all of the same notation such that if they are all consistent, then each is stronger than all its predecessors and therefore translatable into none of them. There exists also a sequence of predicates $\mathrm{P}_{1}, \mathrm{P}_{2}, \cdots$ such that $\mathrm{P}_{m}$ is definable in $\mathrm{L}_{n}$ when and only when $m$ is not greater than $n$.

Using the notion of translation, Gödel's $\left({ }^{18}\right)$ theorem on the incompleteness of systems can also be stated:

3.5. There exists no consistent system $S$ such that there is a translation of the number theory $Z$ into $S$ according to which, for every statement $A$ of $Z$, either $\mathrm{A}$ or $-\mathrm{A}$ is translated into a theorem of $\mathrm{S}$.

HARVARD UNIVERSITY,

CAMBRidge, Mass.

(17) For example, it is sufficient to take $Z_{T_{2}}, Z_{T_{4}}, Z_{T_{6}}, \cdots$ as $L_{1}, L_{2}, L_{3}, \cdots$ where $T_{i}$ is again the $i$ th order predicate calculus.

(18) This corresponds rather to Rosser's extension of Gödel's theorem. See J. Symbolic Logic vol. 1 (1936) pp. 87-91. 